\title{
Rings of congruence preserving functions
}

\author{
C. J. Maxson $^{1}$ - Frederik Saxinger ${ }^{2}$ (D)
}

Received: 28 July 2017 / Accepted: 5 October 2017 / Published online: 8 November 2017

(C) The Author(s) 2017. This article is an open access publication

\begin{abstract}
Let $C_{0}(G)$ denote the near-ring of congruence preserving functions of the group $G$. We investigate the question "When is $C_{0}(G)$ a ring?". We obtain information externally via the lattice structure of the normal subgroups of $G$ and internally via structural properties of the group $G$.
\end{abstract}

Keywords Congruence preserving functions $\cdot 1$-affine complete $\cdot$ Normal subgroup lattice

Mathematics Subject Classification 08A30 $\cdot 16$ Y30 $\cdot 20$ E15

\section{Introduction: background and notation}

Let $G=\langle G,+, 0\rangle$ be a group, written additively but not necessarily abelian, with neutral element 0 . The structure of the near-ring $C_{0}(G)=\left\langle C_{0}(G),+\right.$, ○ $\rangle$ of zero fixing congruence preserving functions on $G$ has been the topic of several previous

Communicated by A. Constantin.

Otto-Herschmann-Gasse 4/4/23, 1110 Vienna, Austria.

$凶$ Frederik Saxinger

frederik.saxinger@yahoo.de

C. J. Maxson

cjmaxson@math.tamu.edu

1 Department of Mathematics, Texas A\&M University, College Station, TX 77843-3368, USA

2 Institute for Algebra, Johannes Kepler Universität-Linz, 4040 Linz, Austria 
investigations $[1,4]$. In this paper we initiate the study of characterizing those groups $G$ such that $C_{0}(G)$ is a ring.

This investigation also has roots in universal algebra. Recall that a unary polynomial function $p: G \rightarrow G$ is a function that can be written in the form $p(x):=a_{0}+k_{0} x+$ $a_{1}+k_{1} x+\cdots+a_{n-1}+k_{n-1} x+a_{n}, x \in G, n$ a nonnegative integer, $a_{0}, a_{1}, \ldots, a_{n} \in$ $G, k_{0}, \ldots, k_{n-1} \in \mathbb{Z}$. We let $P_{0}(G)=\left\langle P_{0}(G),+\right.$, o $\rangle$ denote the near-ring of zero preserving polynomial functions on $G$ (See $[1,11]$.)

The near-ring $C_{0}(G)$ is a subnear-ring of the near-ring $M_{0}(G):=\{f: G \rightarrow G \mid$ $f(0)=0\}$ of zero fixing self maps on $G$ where, as usual, the operations in $M_{0}(G)$ are pointwise addition of functions and composition of functions. Let Inn $(G)$ denote the semigroup (under composition) of inner automorphisms of the group $G$ and let $I(G)$ denote the subnear-ring of $M_{0}(G)$ generated additively by $\operatorname{Inn}(G)$. From the definitions we see $P_{0}(G)=I(G)$.

Now let $f \in C_{0}(G)$. Then by definition, for each congruence, $\rho$ of $G$, for each $x, y \in G, x \rho y$ implies $f(x) \rho f(y)$. As is well-known, there is a lattice isomorphism between the congruence lattice, $\operatorname{Con}(G)$, of congruences on $G$ and the lattice, $\eta(G)$, of normal subgroups of $G$. Thus $f \in M_{0}(G)$ is congruence preserving if $f$ is compatible with every normal subgroup of $G$. That is for $x, y \in G$ we have $f \in C_{0}(G)$ if and only if $x+H=y+H$ implies $f(x)+H=f(y)+H$, for each $H \in \eta(G)$.

Recall for any subgroup $H$ of $G$ the normal closure of $H$ in $G$, denoted by $\bar{H}$, is defined by $\bar{H}=\bigcap\{N \unlhd G \mid H \subseteq N\}$. For $x \in G$ we write $\bar{x}$ for $\overline{\langle x\rangle}$, the principal closure of $x$. Thus we get the following characterization of congruence preserving functions: Let $f \in M_{0}(G)$. Then $f \in C_{0}(G)$ if and only if $f(x)-f(y) \in \overline{x-y}$ for each $x, y \in G$.

Using the above definitions one finds that every zero fixing unary polynomial on $G$ is congruence preserving so $I(G)=P_{0}(G) \subseteq C_{0}(G) \subseteq M_{0}(G)$. If every congruence preserving function is also a polynomial then the group $G$ is said to be 1-affine complete. Finite abelian 1-affine complete groups have been characterized by Nöbauer [14]. All 1-affine complete groups of order up to 100 can be found in [17]. In relation to our problem under consideration, $C_{0}(G)$ will be a ring if $P_{0}(G)$ is a ring and $G$ is 1-affine complete.

It is known when $I(G)=P_{0}(G)$ is a ring [8]. A group $G$ is said to be a 2-Engel group if $[x,[x, y]]=0$ for all $x, y \in G$. Equivalently (see $[8,12,13]) G$ is a 2-Engel group if every element of $G$ commutes with all of its conjugates. A 2-Engel group $G$ is nilpotent of class at most 3 and, for finite groups, a 2-Engel group of class 3 must be a group of order $3^{n}$. The smallest 2-Engel group of class 3 is the Burnside group, $B(3,3)$, of order $3^{7}$.

Theorem 1.1 (Chandy) The near-ring $I(G)$ is a ring if and only if $G$ is a 2-Engel group. Moreover, $I(G)$ is a commutative ring if and only if $G$ is of class 2.

In the remainder of this paper we restrict to finite groups $G$. In the next section we first show when considering $C_{0}(G)$ we may restrict to $p$-groups, $p$ a prime. Using results of Nöbauer [14] we completely characterize those finite abelian groups $G$ such that $C_{0}(G)$ is a ring.

In Sect. 3 we turn to nonabelian groups and find several necessary conditions for $C_{0}(G)$ to be a ring. In this section we focus on certain properties of the lattice 
$\eta(G)$ of normal subgroups. In the final section we consider internal properties of the group $G$ and we conclude with a complete answer to our question for groups of order $p^{n}, 1 \leq n \leq 5, p>2$.

\section{The abelian case}

We begin this section by showing that, for finite groups, we can restrict to $p$-groups, where as usual $p$ denotes a prime integer. We use results of Nöbauer, [14], to obtain this restriction.

We recall from [14] that a direct sum $G=G_{1} \oplus \cdots \oplus G_{n}$ is said to be skew-free if every congruence $\rho$ of $G$ is of the form $\rho=\rho_{1}+\cdots+\rho_{n}$ where the $\rho_{i}$ are congruences on the $G_{i}, i=1,2, \ldots, n$. In particular when $G$ is a finite nilpotent group with direct sum of its unique Sylow subgroups $G=S_{p_{1}}(G) \oplus \cdots \oplus S_{p_{n}}(G)$ then $G$ is skew-free. Recall from the introduction that $\operatorname{Con}(G)$ is lattice isomorphic to $\eta(G)$, the lattice of normal subgroups of $G$.

Theorem 2.1 [14, Satz 1] Let $G=A \oplus B$ be skew-free. Then the map $\psi: C_{0}(G) \rightarrow$ $C_{0}(A)+C_{0}(B)$ given by $\psi(\rho)=\left(\rho_{A}, \rho_{B}\right), \rho \in C_{0}(G)$ is a near-ring isomorphism.

From straight forward calculations one finds the following theorem and corollary.

Theorem 2.2 Let $G$ be a finite nilpotent group, $G=S_{p_{1}}(G) \oplus \cdots \oplus S_{p_{n}}(G)$. Then $C_{0}(G) \cong C_{0}\left(S_{p_{1}}(G)\right) \oplus \cdots \oplus C_{0}\left(S_{p_{n}}(G)\right)$.

Corollary 2.3 Let $G$ be a finite group with Sylow decomposition as in Theorem 2.2. Then $C_{0}(G)$ is a ring if and only if $C_{0}\left(S_{p_{i}}(G)\right)$ is a ring for each $i \in\{1,2, \ldots, n\}$.

Hence we only need to consider groups, $G$, of prime power order when investigating the structure of the near-ring, $C_{0}(G)$, of zero fixing congruence preserving functions on $G$.

We now turn to abelian groups. We need a further result of Nöbauer [14].

Theorem $2.4\left[14\right.$, Satz 3,4] Let $p$ be a prime, $\mathbb{Z}_{p^{\alpha}}$ be the cyclic group of order $p^{\alpha}$ and let $A=\mathbb{Z}_{p^{\alpha_{1}}} \oplus \cdots \oplus \mathbb{Z}_{p^{\alpha_{n}}}$ with $\alpha_{1} \geq \alpha_{2} \geq \cdots \geq \alpha_{n}$. Then $A$ is 1-affine complete if and only if one of the following conditions holds:

(a) $n>1, \alpha_{1}=\alpha_{2}, p$ arbitrary,

(b) $n>1, \alpha_{1}=\alpha_{2}+1, p=2$ or

(c) $n=1, \alpha_{1}=1, p=2$.

Theorem 2.5 Let $A$ be an abelian group of prime power order. The following are equivalent:

(1) A is 1-affine complete;

(2) $C_{0}(A)$ is a ring;

(3) $C_{0}(A)$ is a commutative ring.

Proof Since $A$ is abelian, $A$ is a 2-Engel group of nilpotency class at most 2 so $I(A)=P_{0}(A)$ is a commutative ring. If $A$ is 1 -affine complete then $C_{0}(A)$ is a ring. Thus (1) implies (3). 
It is clear that (3) implies (2) so it remains to show (2) implies (1). We assume $A$ is not 1 -affine complete and show $C_{0}(A)$ is not a ring. From Theorem 2.4 we know the form $A$ has to be so that $A$ is not 1-affine complete.

Case (i) $|A|=p^{m}$ for some prime $p>2$. In this case we must have $A \cong \mathbb{Z}_{p^{\alpha_{1}}} \oplus$ $\cdots \oplus \mathbb{Z}_{p^{\alpha_{n}}}$ with $n=1$ or $\alpha_{1}>\alpha_{2} \geq \cdots \geq \alpha_{n}$. We let $\mathbb{Z}_{p^{\alpha_{1}}}=\langle g\rangle$ and let $D=\left\{x \in A \mid p^{\alpha_{1}-1} x=0\right\}=\left\{\left(a_{1}, \ldots, a_{n}\right) \mid a_{1} \in\langle p g\rangle\right\}$.

Define $c: A \rightarrow A$ by

$$
c(x)= \begin{cases}(0, \ldots, 0), & x \in D \\ \left(p^{\alpha_{1}-1} g, 0, \ldots, 0\right), & x \notin D .\end{cases}
$$

We show $c \in C_{0}(A)$. To this end, let $x, y \in A$. If $x, y \in D$ or $x, y \notin D$ then $c(x)-c(y)=(0, \ldots, 0) \in \overline{x-y}$ so we take $x \notin$ $D, y \in D$. Thus $c(x)-c(y)=\left(p^{\alpha-1} g, 0, \ldots, 0\right)$. Now $p^{\alpha_{1}-1}(x-y)=$ $\left(p^{\alpha_{1}-1} k g, 0, \ldots, 0\right), p \nmid k$ so $x \notin D$. But then $\left(p^{\alpha_{1}-1} g, 0, \ldots, 0\right) \in \overline{x-y}$ so $c \in C_{0}(A)$. Further, $[c(\mathrm{id}+\mathrm{id})]((g, 0, \ldots, 0))=c(\operatorname{id}((g, 0, \ldots, 0))+$ $\operatorname{id}((g, 0, \ldots, 0)))=c((2 g, 0, \ldots, 0))=\left(p^{\alpha_{1}-1} g, 0, \ldots, 0\right)$ while $[c \circ$ $\mathrm{id}+c \circ \mathrm{id}]((g, 0, \ldots, 0))=2 c((g, 0, \ldots, 0))=2\left(p^{\alpha_{1}-1} g, 0, \ldots, 0\right) \neq$ $\left(p^{\alpha_{1}-1} g, 0, \ldots, 0\right)$. Hence $C_{0}(G)$ is not a ring.

Case (ii) $|A|=2^{m}$. In this case $A=\mathbb{Z}_{2^{\alpha_{1}}} \oplus \cdots \oplus \mathbb{Z}_{2^{\alpha_{n}}}$ with either $n>1$ and $\alpha_{1}-1>\alpha_{2} \geq \cdots \geq \alpha_{n}$ or $n=1$ and $\alpha_{1}>1$. Again we handle both cases together and as above we let $\mathbb{Z}_{2^{\alpha_{1}}}=\langle g\rangle$. Let $D=\left\{x \in A \mid 2^{\alpha_{1}-2} x=0\right\}=$ $\left\{\left(a_{1}, \ldots, a_{n}\right) \mid a_{1} \in\langle 4 g\rangle\right\}$ and further let $\bar{D}=D \cup(D+(2 g, 0, \ldots, 0))$. Define $c: A \rightarrow A$ by

$$
c(x)= \begin{cases}(0,0, \ldots, 0), & x \in \bar{D} \\ \left(2^{\alpha_{1}-2} g, 0, \ldots, 0\right), & x \notin \bar{D}\end{cases}
$$

As above we show $c \in C_{0}(A)$. Let $x, y \in A, x \notin \bar{D}, y \in \bar{D}$. Then $c(x)-c(y)=\left(2^{\alpha_{1}-2} g, 0, \ldots, 0\right)$. Now since $x \notin \bar{D}, x=$ $(k g, 0, \ldots, 0)$ where $k \in\{1,3\}$. Thus $2^{\alpha_{1}-2}(x-y)=\left(2^{\alpha_{1}-2} k g, 0, \ldots, 0\right)$ so $\left(2^{\alpha_{1}-2} g, 0, \ldots, 0\right) \in \overline{x-y}$. Using $f=\mathrm{id}, h=3 \cdot$ id we have $[c(f+$ $g)]((g, 0, \ldots, 0))=[c(\mathrm{id}+3 \cdot \mathrm{id})]((g, 0, \ldots, 0))=c(\mathrm{id}((g, 0, \ldots, 0))+$ $3 \cdot \operatorname{id}((g, 0, \ldots, 0)))=c((4 g, 0, \ldots, 0))=(0, \ldots, 0) \neq c((g, 0, \ldots, 0))+$ $c((3 g, 0, \ldots, 0))$. So $C_{0}(A)$ is not a ring.

We now have a characterization of those finite abelian groups $A$ for which $C_{0}(A)$ is a ring.

Corollary 2.6 Let A be a finite abelian group. The following are equivalent:

(1) A is 1-affine complete;

(2) $C_{0}(A)$ is a ring;

(3) $C_{0}(A)$ is a commutative ring. 
Proof As in Theorem 2.5 (1) implies (3) and (3) implies (2). Using the Sylow decomposition of $A$ we see that (2) implies (1) follows from Theorem 2.5 and Nöbauer [14, Lemma 5].

In the next section we give several necessary conditions for $C_{0}(G)$ to be a ring.

\section{Lattice conditions}

We start with some conditions on the congruence lattice, $\operatorname{Con}(G)$. Since $\operatorname{Con}(G)$ is lattice isomorphic to the normal subgroup lattice, $\eta(G)$, we often state our properties in terms of normal subgroups.

We recall that $G$ must be a 2-Engel group, and thus nilpotent of class at most 3, for $C_{0}(G)$ to be a ring.

Our first lattice concept is that of splitting pair. This property has been used previously [3,5,15]. Let $D, E \in \eta(G), D \subset G,\{0\} \subset E$. The pair $(D, E)$ is called a splitting pair if for each $N \in \eta(G), N \subseteq D$ or $N \supseteq E$. If $G$ has a splitting pair then $G$ splits.

Now let $(D, E)$ be a splitting pair for $G$ and let $0 \neq b \in E$. Define $f: G \rightarrow G$ by

$$
f(x)= \begin{cases}0, & x \in D \\ b, & \text { otherwise }\end{cases}
$$

We show $f \in C_{0}(G)$. Let $x, y \in G$ and let $H \in \eta(G)$ with $x+H=y+H$. If $E \subseteq H$ then since $f(x)-f(y) \in\{-b, 0, b\} \subseteq E$ we get $f(x)+H=f(y)+H$. If $H \subseteq D$ and $x \in D$ then $x+H \subseteq D$ and so $y+H \subseteq D$ which means $y \in D$. By symmetry if $x \notin D$ then $y \notin D$, hence in both cases $f(x)+H=f(y)+H$. This establishes that $f \in C_{0}(G)$. Now if $C_{0}(G)$ is a ring then for $v \notin D,[f \circ(\mathrm{id}+\mathrm{id})](v)=$ $[(f \circ \mathrm{id}+f \circ \mathrm{id})](v)$ or $f(2 v)=2 f(v)$. If $2 v \notin D$ then $b=2 b$, which contradicts $b \neq 0$. Therefore $2 v \in D$ and further $0=2 b$. Since $0 \neq b$ was arbitrary in $E$ we get $E \cong\left(\mathbb{Z}_{2}\right)^{n}$ for some $n>0$. This establishes:

Theorem 3.1 Let $G$ be a finite group such that $2 \nmid|G|$. If $G$ splits then $C_{0}(G)$ is not a ring.

Proof From the above discussion, when $G$ splits then $G$ has a subgroup $E \cong$ $\left(\mathbb{Z}_{2}\right)^{n}, n>0$ which is a contradiction since $2 \nmid|G|$.

In particular if $G$ is a finite $p$-group, $p>2$, and $G$ splits, then $C_{0}(G)$ is not a ring. The situation is different in the non-split case as the next examples illustrate. These examples and some of the calculations have been done with GAP using the package Sonata [2].

Example 3.2 (1) Group with GAP index $3^{7} / 6010 . G=\left\langle e_{1}, e_{2}, e_{3}, e_{4}, c_{1}, c_{2}, c_{3}\right\rangle, 3 e_{i}$ $=3 c_{j}=0,\left[e_{i}, c_{j}\right]=\left[c_{k}, c_{j}\right]=0,\left[e_{1}, e_{2}\right]=c_{1},\left[e_{1}, e_{3}\right]=c_{2},\left[e_{2}, e_{3}\right]=c_{3}$ otherwise $\left[e_{l}, e_{m}\right]=0, i=1,2,3,4, j, k=1,2,3$. Thus $G$ is a group of exponent 3, nilpotent of class 2 with $G^{\prime}=\left\langle c_{1}, c_{2}, c_{3}\right\rangle \subseteq Z(G)$ [9]. From GAP, $G$ does not split but is 1-affine complete so $C_{0}(G)$ is a ring since nilpotent of class 2 means $G$ is 2-Engel. 
(2) GAP index $3^{7} / 6576 . G=\left\langle e_{1}, e_{2}, e_{3}, e_{4}, c_{1}, c_{2}, c_{3}\right\rangle, 3 e_{i}=3 c_{i}=0,\left[e_{i}, c_{j}\right]=$ $\left[c_{k}, c_{j}\right]=0, i=1,2,3,4, j, k=1,2,3$ with $\left[e_{1}, e_{2}\right]=c_{1},\left[e_{1}, e_{3}\right]=$ $\left[e_{2}, e_{4}\right]=c_{2},\left[e_{3}, e_{4}\right]=c_{3}$, otherwise $\left[e_{m}, e_{l}\right]=0$. Again $G$ is of exponent 3 , nilpotent of class 2 , with $G^{\prime}=\left\langle c_{1}, c_{2}, c_{3}\right\rangle \subseteq Z(G)$ [9]. Using GAP, $G$ does not split and is not 1-affine complete. We show $C_{0}(G)$ is not a ring. For $x \in G, x=\alpha e_{1}+\beta e_{2}+\gamma e_{3}+\delta e_{4}+d$ where $d \in G^{\prime}$. Define $f: G \rightarrow G$ by

$$
f(x)= \begin{cases}2\left(c_{1}+c_{2}+c_{3}\right), & \text { if } \beta+\gamma=3, \\ 2 c_{3}, & \text { if } \beta=0, \gamma \neq 0, \\ 2 c_{1}, & \text { if } \beta \neq 0, \gamma=0, \\ 2 c_{1}+c_{2}+2 c_{3}, & \text { if } \beta=\gamma \neq 0, \\ 0, & \text { otherwise. }\end{cases}
$$

Calculations show that $f \in C_{0}(G)$. Now $f \circ(\mathrm{id}+\mathrm{id})\left(e_{3}\right)=f\left(2 e_{3}\right)=2 c_{3}$ while $(f \circ \mathrm{id}+f \circ \mathrm{id})\left(e_{3}\right)=2 c_{3}+2 c_{3}=c_{3} \neq 2 c_{3}$. Thus $C_{0}(G)$ is not a ring.

Above we denoted the normal closure of $x \in G$ by $\bar{x}$. For $x \in G$, let $P_{0}(G) x=$ $\left\{p(x) \mid p \in P_{0}(G)\right\}$ and $C_{0}(G) x=\left\{c(x) \mid c \in C_{0}(G)\right\}$.

Lemma 3.3 Let $G$ be a group and let $x \in G$.

(1) $P_{0}(G) x=\bar{x}$.

(2) $P_{0}(G) x=C_{0}(G) x$.

If further $G$ is 2-Engel then

(3) $\bar{x}$ is abelian;

(4) $\left\langle C_{0}(G),+\right\rangle$ is an abelian group.

(5) If $G$ is nilpotent of class at most 2 then $\bar{x}=\langle x\rangle+[x, G]$.

Proof (1) Clearly $P_{0}(G) x \subseteq \bar{x}$. On the other hand, $P_{0}(G) x$ is a normal subgroup of $G$ containing $x$, so $\bar{x} \subseteq P_{0}(G) x$.

(2) One has $P_{0}(G) x \subseteq C_{0}(G) x$. For $c \in C_{0}(G), c(x)-c(0) \in \overline{x-0}$ so $c(x) \in \bar{x}$. Thus $C_{0}(G) x \subseteq \bar{x}=P_{0}(G) x$.

(3) When $G$ is 2-Engel, $P_{0}(G)$ is a ring so with 1) we get that $P_{0}(G) x$ is an abelian group.

(4) Follows from 2) since $G$ is 2-Engel.

(5) In [10] Ecker shows $p \in P_{0}(G)$ has the form $p(x)=k x+[x, g]$ for some integer $k$ and $g \in G$ when $G$ is nilpotent of class at most 2. Thus $P_{0}(G) x \subseteq\langle x\rangle+[x, G]$. But $\langle x\rangle+[x, G] \subseteq \bar{x}=P_{0}(G) x$.

We next give a characterization of those groups $G$ for which $C_{0}(G)$ is a ring. The usefulness of this result is somewhat limited since it requires knowledge of all $c \in C_{0}(G)$.

Theorem 3.4 Let $G$ be a finite 2-Engel group. Then $C_{0}(G)$ is a ring if and only if $\left.c\right|_{\bar{x}} \in \operatorname{End}(\bar{x})$ for each $c \in C_{0}(G)$ and $x \in G$.

Proof Let $c, f, g$ be arbitrary in $C_{0}(G)$ and let $x \in G$. From Lemma $3.3\left\langle C_{0}(G),+\right\rangle$ is an abelian group. Suppose $\left.c\right|_{\bar{x}} \in \operatorname{End}(\bar{x})$ for each $x \in G$. Then $c \circ(f+g)(x)=$ 
$c(f(x)+g(x))=c(f(x))+c(g(x))=(c \circ f+c \circ g)(x)$, since $f(x), g(x) \in \bar{x}$ by (1) and (2) of Lemma 3.3. Thus $C_{0}(G)$ is a ring.

For the converse let $a, b \in \bar{x}$. Thus by 1) and 2) of Lemma 3.3 there exist $h, l \in$ $C_{0}(G), a=h(x), b=l(x)$. Now let $c \in C_{0}(G)$. It is clear that $c(\bar{x}) \subseteq \bar{x}$. Moreover since $C_{0}(G)$ is a ring we have $c(a+b)=c(h(x)+l(x))=c(h(x))+c(l(x))=$ $c(a)+c(b)$ which shows $c \in \operatorname{End}(\bar{x})$.

Therefore if one can construct a congruence preserving function that is not linear on some normal closure $\bar{x}, x \in G$, where $G$ is 2-Engel, then $C_{0}(G)$ is not a ring. In the next example, using Theorem 3.4, we show that the result of Theorem 3.1 on groups which split is not true for $p=2$.

Example 3.5 Let $G$ be a semidirect product of $\mathbb{Z}_{4}$ and $\mathbb{Z}_{4}: G=\langle x, y| 4 x=4 y=$ $0, y+x=3 x+y\rangle$. We have $Z(G)=\langle 2 x, 2 y\rangle$ and one verifies that $D=Z(G)$ and $E=\langle 2 x\rangle$ is a splitting pair for $\eta(G)$. Define $c: G \rightarrow G$ by

$$
c(w)= \begin{cases}0, & w \in Z(G), \\ 2 x, & w \notin Z(G) .\end{cases}
$$

Let $u, v \in G$. If $u, v \in Z(G)$ or $u, v \notin Z(G)$ then $c(u)-c(v)=0 \in \overline{u-v}$. If $u \notin Z(G)$ and $v \in Z(G)$ then $u-v \notin Z(G)$ and so $\overline{u-v} \supseteq\langle 2 x\rangle$ since $(Z(G),\langle 2 x\rangle)$ is a splitting pair. Thus $c(u)-c(x)=2 x \in \overline{u-v}$ so $c \in C_{0}(G)$ but $c \notin P_{0}(G)$ since $c(x)=2 x$ while $c(y)=2 x \neq 2 y$.

Using GAP one finds $\left|P_{0}(G)\right|=16$ and $\left|C_{0}(G)\right|=32$ so we have $C_{0}(G)=$ $P_{0}(G)+\langle c\rangle=\left\{p+l c \mid l \in\{0,1\}, p \in P_{0}(G)\right\}$. For $w \notin Z(G)$, calculations show that $c$ is linear on $\bar{w}$. Thus for all $w \in G,\left.c\right|_{\bar{w}} \in \operatorname{End}(\bar{w})$. Thus for each $p \in P_{0}(G), p+c$ is linear on each $\bar{w}$ so from Theorem $3.4 C_{0}(G)$ is a ring.

We turn to another lattice condition, a particular case of a splitting pair. If $(D, E)$ is a splitting pair for $\eta(G)$ and $D=E$ we say $D$ is a cutting element and $G$ cuts.

Lemma 3.6 Let $G$ be a finite p-group of nilpotency class at most 2 such that $I$ is a cutting element for $\eta(G)$. Then $I \subseteq Z(G)$.

Proof Let $T$ be the maximal cutting element for $\eta(G)$ which exists since $G$ is finite and cutting elements form a chain in $\eta(G)$. We have $T \supseteq I$. If $G$ is abelian then $I \subseteq Z(G)=G$ so we take $G$ of class 2 , hence $G^{\prime} \subseteq Z(\bar{G})$. If $T$ is also a maximal element in $\eta(G)$ then $G$ has a unique maximal normal subgroup. Thus from [16], $G$ is cyclic, contrary to $G$ being of class 2 . Thus we suppose $T$ is not a maximal element in $\eta(G)$. If $T \subseteq G^{\prime}$ then $T$, hence $I$, is contained in $Z(G)$. To complete the proof we show $G^{\prime} \subset T$ cannot occur. Suppose $G^{\prime} \subset T$ and let $N \in \eta(G)$ be maximal with $G^{\prime} \subseteq N \subset T$. Since $G / G^{\prime}$ is abelian, $G / N$ is also abelian. Therefore $G / N$ has a unique minimal normal subgroup $T / N$. But this means that $G / N$ is subdirectly irreducible and (from [7] p. 64) $G / N$ is cyclic. However this contradicts the fact that $T$ is the unique maximal cutting element but not a maximal element in $\eta(G)$. Thus we have $I \subseteq Z(G)$. 
Theorem 3.7 Let $G$ be a finite nonabelian p-group such that $G$ cuts. Then $C_{0}(G)$ is not a ring.

Proof Let $I$ be a cutting element. If $G$ is of nilpotency class greater than 3 then $G$ is not 2-Engel, hence $C_{0}(G)$ is not a ring. Further if $p>2$ then from Theorem 3.1, $C_{0}(G)$ is not a ring. Therefore $p=2$ and $G$ is nilpotent of class $2,|G|=2^{n}, n \geq 3$. From Lemma 3.6 we get $I \subseteq Z(G)$.

Let $T_{1}$ be a transversal of $G / I$ with $0 \in T_{1}$. Let $t \in T_{1}-\{0\}$ and define $T_{2}=$ $\left(T_{1} \backslash(t+t+I)\right) \cup\{t+t\}$. We note $T_{2}$ is a transversal of $G / I$ with $\{t, t+t\} \subseteq T_{2}$. Suppose first that $t+t \notin I$ so $0 \in T_{2}$. Let $0 \neq e$ be in $I$ and define $h: G \rightarrow G$ by

$$
h(x)= \begin{cases}e, & x \in t+t+I, \\ 0, & \text { otherwise }\end{cases}
$$

We note that $h(0)=0$. To show $h \in C_{0}(G)$ it suffices to show for $r \in T_{2}-\{t+$ $t\}, d_{1}, d_{2} \in I$ that $h\left(t+t+d_{1}\right)-h\left(r+d_{2}\right) \in \overline{t+t+d_{1}-d_{2}-r}$, that is, we must show $e \in \overline{t+t+d_{1}-d_{2}-r}$. We first observe that $t+t+d_{1}-d_{2}-r=$ $t+t-r+d_{1}-d_{2} \notin I$ since $r \in T_{2}-\{t+t\}$. Therefore $\overline{t+t+r+d_{1}-d_{2}} \nsubseteq I$ and, since $I$ cuts $\eta(G), I \subseteq \overline{t+t+r+d_{1}-d_{2}}$ giving the desired result that $h \in C_{0}(G)$. But $h(t+t)=e \neq h(t)+h(t)$. Since $h$ is not linear on $\bar{t}, C_{0}(G)$ is not a ring.

Suppose next we have $0 \neq t+t \in I$. Using $T_{1}$ we define $f: G \rightarrow G$ by $f(x)=j$ where $x=r+j, r \in T_{1}, j \in I$. Since $0 \in T_{1}, f(0)=0$. For $x=r_{1}+j_{1}, y=$ $r_{2}+j_{2}, f(x)-f(y)=j_{1}-j_{2}$. If $r_{1}=r_{2}, j_{1}-j_{2} \in \overline{r_{1}-r_{2}+j_{1}-j_{2}}$. If $r_{1} \neq r_{2}$ then $r_{1}-r_{2}+j_{1}-j_{2} \notin I$ so $I \subseteq \overline{r_{1}-r_{2}+j_{1}-j_{2}}$, hence $f(x)-f(y)=j_{1}-j_{2} \in \overline{x-y}$. Thus $f \in C_{0}(G)$. Since $t+t \in I, t+t=0+t+t$ which means $f(t+t)=t+t$. But $t=t+0$ so $f(t)=0=f(t)+f(t) \neq f(t+t)$. This shows that $C_{0}(G)$ is not a ring.

For the final case we have $t+t \in I$ and $t+t=0$. Define $l: G \rightarrow G$ by

$$
l(x)= \begin{cases}0, & x \in I, \\ j, & x \notin I \text { and } x=r+j, r \in T_{2}=T_{1} .\end{cases}
$$

For $x \notin I, y \in I$ say $x=r+j, r \in T_{2}, j \in I$ we have $l(x)-l(y)=j$. Moreover $I \subseteq \overline{r+j-y}$ since $r+j-y \notin I$. Thus $l \in C_{0}(G)$. Since $t+t \in I$ for any $0 \neq i \in I, l(t+t+i)=0$. Further since $t \notin I, I \subseteq \bar{t}$, hence $t+i \in \bar{t}$. Now $l(t)+l(t+i)=0+i \neq 0$ so $l$ is not linear on $\bar{t}$. Thus in all cases we have found when $G$ is cut, $C_{0}(G)$ is not a ring.

\section{Structural conditions}

In this section we focus on group theoretical properties of a group $G$ to determine when $C_{0}(G)$ is a ring. We restrict to nilpotency class 2 and $p$-groups $p \geq 3$.

Theorem 4.1 Let $G$ be a nonabelian p-group, $p>2$ such that $G^{\prime}$ is cyclic. Then $C_{0}(G)$ is not a ring. 
Proof Let $x \in G-Z(G)$. Thus $\{0\} \neq[x, G] \subseteq[G, G]$. By hypothesis, $G^{\prime}=\langle r\rangle$ for some $r \in G$ so we have $\bar{r}=\langle r\rangle=G^{\prime}$.

Let $\left\langle r^{\prime}\right\rangle$ be the unique subgroup of order $p$ in $G^{\prime}$. Since $[x, G]$ is a nonzero cyclic subgroup of $G^{\prime},\left[x, G^{\prime}\right]$ contains a cyclic subgroup of order $p$ of $G^{\prime}$, hence we have $\left\langle r^{\prime}\right\rangle$ is a subgroup of $\left[x, G^{\prime}\right]$. Moreover, for each $g \in G, p\left(-g+r^{\prime}+g\right)=0$ so $-g+r^{\prime}+g$ is in $\left\langle r^{\prime}\right\rangle$ which in turn leads to the fact that $\left\langle r^{\prime}\right\rangle$ is a normal subgroup of $[x, G]$. Therefore, for $x \in G-Z(G), \overline{r^{\prime}}=\left\langle r^{\prime}\right\rangle \subseteq[x, G] \subseteq \bar{x}$.

Define $h: G \rightarrow G$ by

$$
h(x)= \begin{cases}r^{\prime}, & x \notin Z(G), \\ 0, & x \in Z(G) .\end{cases}
$$

We show $h \in C_{0}(G)$. To this end let $u \notin Z(G), v \in Z(G)$. Then $h(u)-h(v)=r^{\prime}$ which is in $\overline{u-v}$ since $u-v \notin Z(G)$. This gives $h \in C_{0}(G)$. For $w \notin Z(G), 2 w=$ $w+w \notin Z(G)$ since $p>2$ so if $2 w \in Z(G)$, we would have $w \in Z(G)$, a contradiction. From this observation, $h(w+w)=r^{\prime} \neq 2 r^{\prime}=h(w)+h(w)$. Therefore $C_{0}(G)$ is not a ring.

We actually have a little more.

Corollary 4.2 If $G$ is a nonabelian p-group, $p>2$, such that $G^{\prime}$ is cyclic, then $\eta(G)$ splits.

Proof From the proof of Theorem 4.1 we get if $N \unlhd G$ and $N \nsubseteq Z(G)$ then for $x \in N-Z(G), N \supseteq \bar{x} \supseteq \overline{r^{\prime}}$. Thus $\left\langle Z(G), \overline{r^{\prime}}\right\rangle$ is a splitting pair for $\eta(G)$.

Corollary 4.3 Let $G$ be a p-group, $p>2$, of nilpotency class 2. If $G$ is 2-generated (generated by 2 elements) then $C_{0}(G)$ is not a ring.

Proof If $G=\langle x, y\rangle$ then one finds $G^{\prime}=\langle[x, y]\rangle$ so $G^{\prime}$ is cyclic [6]. The result now follows from the above theorem.

We remark that Example 3.5 shows that Corollary 4.3 does not hold for $p=2$.

Recall that a group $G$ is abelian by cyclic, or $G$ is said to be an extension of an abelian group by a cyclic group if there exists an abelian normal subgroup $A$ of $G$ such that $G / A$ is cyclic. For finite $G$ one may always take $A$ to be a maximal abelian normal subgroup.

Theorem 4.4 Let $G$ be a nonabelian p-group, $p>2$, of nilpotency class 2 which is abelian by cyclic. Then $C_{0}(G)$ is not a ring.

Proof We let $A$ be a maximal abelian normal subgroup such that $G / A \cong \mathbb{Z}_{p^{k}}, k$ a positive integer. Let $G / A=\langle b+A\rangle$ so $G=\langle A, b\rangle$. Since $G$ is nonabelian there exists $a_{1} \in A$ such that $\left[a_{1}, b\right] \neq 0$. Every $x \in G$ can be decomposed into a sum of the form $x=a+\beta b+c$, with $a \in A, \beta \in \mathbb{Z}, c \in G^{\prime}$. (Recall the basic assumption that $G$ is of class 2 so $[G, G] \subseteq Z(G)$.) 
Using this decomposition we define $f: G \rightarrow G$ by

$$
f(x)= \begin{cases}{\left[b, a_{1}\right],} & p \nmid \beta, \\ 0, & p \mid \beta .\end{cases}
$$

Let $x \in G$. We note $\left[x, a_{1}\right]=\beta\left[b, a_{1}\right]$ so when $p \nmid \beta,\left[b, a_{1}\right] \in \bar{x}$. Also, $f \in C_{0}(G)$. For if $u, v \in G, u=a+\beta b+c, v=a^{\prime}+\beta^{\prime} b+c^{\prime}, a, a^{\prime} \in A, \beta, \beta^{\prime} \in \mathbb{Z}, c, c^{\prime} \in$ $[G, G]$ with $p \nmid \beta$ and $p \mid \beta^{\prime}$ then $p \nmid\left(\beta-\beta^{\prime}\right)$ so $\left[b, a_{1}\right] \in \overline{u-v}$. Thus $f(u)-f(v)=$ $\left[b, a_{1}\right] \in \overline{u-v}$. For $u=a+\beta b+c$ with $p \nmid \beta, f(u)+f(u)=\left[b, a_{1}\right]+\left[b, a_{1}\right]$ while $f(u+u)=f(2 u)=\left[b, a_{1}\right]$. Using Theorem 3.4, $C_{o}(G)$ is not a ring.

Corollary 4.5 Let $G$ be a nonabelian p-group, $p>2$, of class 2 such that there exists $g \in G$ with $G / C_{G}(g)$ cyclic. Then $C_{0}(G)$ is not a ring.

Proof Let $G / C_{G}(g)=\left\langle b+C_{G}(g)\right\rangle$ so $G=\left\langle C_{G}(g), b\right\rangle$. For $x \in G, x=w+$ $\beta b+c, w \in C_{G}(g), \beta \in \mathbb{Z}, c \in G^{\prime}$ and since $b \notin C_{G}(g),[b, g] \neq 0$. Now $[x, g]=\beta[b, g]$. The remainder of the proof is as above and is omitted.

As we did following Theorem 4.1, we again show that under the hypothesis of Theorem 4.4, $\eta(G)$ splits.

Theorem 4.6 Let $G$ be a p-group, nilpotent of class 2, which is abelian by cyclic. Then $\eta(G)$ splits.

Proof As above we let $A$ be a maximal abelian normal subgroup with $G / A=\langle b+$ $A\rangle$. Then $G=\langle A, b\rangle$ and $b \notin A$ so there exists $a_{1} \in A,\left[a_{1}, b\right] \neq 0$. Let $A=$ $\left\langle a_{1}, a_{2}, \ldots, a_{n}\right\rangle$ so $G=\left\langle a_{1}, \ldots, a_{n}, b\right\rangle$. Let $A_{0}=\left\langle a_{1}, \ldots, a_{n}, p b\right\rangle$. If $p b=0$ then $A_{0}=A$ and the same type of argument works. We first show that $A_{0}$ is a normal subgroup of $G$. Let $g=a+\beta b+c \in G, a \in A, \beta \in \mathbb{Z}, c \in G^{\prime}$. It suffices to show $-g+p b+g \in A_{0}$. To this end, $-g+p b+g=-c-\beta b-a+p b+a+\beta b+c=$ $-\beta b-a+p b+a+\beta b=-\beta b+p b+[p b, a]+\beta b=p b+[p b, a] \in A_{0}$. Therefore $A_{0} \unlhd G$.

Further $A_{0} \subset G$. For if $A_{0}=G$ then $b \in A_{0}=\left\langle a_{1}, a_{2}, \ldots, a_{n}, p b\right\rangle$. From this, $b=a+\alpha(p b)+c$ where $a \in A$ and $c$ is a sum of commutators. Since $A$ is a maximal abelian normal subgroup we have $Z(G) \subseteq A$ and since $G$ is nilpotent of class 2, $[G, G] \subseteq Z(G) \subseteq A$. Thus $c \in A$. Hence $(1-\alpha p) b \in A$ which implies $b \in A$ since $1-\alpha p$ is invertible modulo $p$, a contradiction. Thus $A_{0} \neq G$.

Now let $N \in \eta(G)$ such that $N \nsubseteq A_{0}$. For $n \in N-A_{0}, n=a+\delta b+c, a \in A, p \nmid$ $\delta, c \in G^{\prime}$, hence $\left[n, a_{1}\right]=\delta\left[b, a_{1}\right]$ and since $p \nmid \delta, 0 \neq\left[b, a_{1}\right] \in \bar{n} \subseteq N$. From this, $\left\langle\left[b, a_{1}\right]\right\rangle \subseteq N$ for each $N \in \eta(G)$ such $N \nsubseteq A_{0}$. This shows that $\left(A_{0},\left\langle\left[b, a_{1}\right]\right\rangle\right)$ is a splitting pair for $\eta(G)$.

In Theorems 4.6 and 4.1 one has the situation where $G$ has a partition $G=X \cup$ $(G-X)$ with the property that $\bigcap\{\bar{u} \mid u \in X\} \neq\{0\}$ and, for each $u \in X$, for each $v \in G-X, u-v \in X$. It is an open question if this condition implies the splitting of $\eta(G)$.

We apply the above results to $p$-groups, $p>2$, of small order. Let $G$ be a group of order $p^{n}, p>2,1 \leq n \leq 5$. When $n=1$ or $n=2, G$ is abelian so $C_{0}(G)$ is a ring 
if and only if $G \cong \mathbb{Z}_{p}+\mathbb{Z}_{p}$. Thus we take $n \geq 3$ and since the abelian case is known from Theorems 2.4 and 2.5 we restrict to nonabelian groups.

Theorem 4.7 Let $G$ be a nonabelian $p$-group, $p>2$ of order $p^{n}, 3 \leq n \leq 5$ such that $G$ is nilpotent of class 2. Then $C_{0}(G)$ is not a ring.

Proof (i) $n=3$. Since $g$ is nonabelian we have $|Z(G)|=p$. Thus $\{0\} \neq G^{\prime} \subseteq$ $Z(G)$, hence $G^{\prime}$ is cyclic and the result follows from Theorem 4.1.

(ii) $n=4$. Let $\Phi(G)$ denote the Frattini subgroup of $G$. We know $G^{\prime} \subseteq \Phi(G)$ and if $|G / \Phi(G)|=p^{k}$ then $G$ is generated by $k$ elements [16]. If $|\Phi(G)|=p$ then $G^{\prime}$ is cyclic while if $|\Phi(G)|=p^{2}$ then $G$ is generated by 2 elements. Using Theorem 4.1 and Corollary 4.3 we see that $C_{0}(G)$ is not a ring. If $|\Phi(G)|=p^{3}$ then $G$ has a unique maximal normal subgroup which cuts $G$. The result now follows from Theorem 3.7.

(iii) $n=5$. If $|\Phi(G)|=p$ or $p^{3}$ or $p^{4}$ then as in the above case we have $C_{0}(G)$ is not a ring. It remains to consider $|\Phi(G)|=p^{2}$. We must have $G^{\prime}=\Phi(G)$ for if $G^{\prime} \neq$ $\Phi(G)$ then $G^{\prime}$ is cyclic and we are finished. Thus we have $G^{\prime}=\Phi(G) \subseteq Z(G)$ since $G$ is of class 2. For $x \in G-Z(G)$ define $\varphi_{x}: G \rightarrow G$ by $\varphi_{x}(w)=[x, w]$. Since $G$ is of class $2, \varphi_{x}$ is an endomorphism of $G$ and $\operatorname{ker} \varphi_{x}=C_{G}(x) \supseteq$ $\langle x\rangle+Z(G)$ while $\operatorname{Im} \varphi_{x}=[x, G] \subseteq \bar{x}$. We have $|\langle x\rangle+Z(G)|=p|Z(G)|$ so if $G^{\prime} \subset Z(G)$ then $|Z(G)|=p^{3}$ and $\left|\operatorname{ker} \varphi_{x}\right|=\left|C_{G}(x)\right|=|\langle x\rangle+Z(G)|=p^{4}$. But this means $G$ is abelian by cyclic so the result follows from Theorem 4.4. Thus we take $\left|G^{\prime}\right|=|\Phi(G)|=|Z(G)|=p^{2}$. Thus $\left|\operatorname{ker} \varphi_{x}\right|=\left|C_{G}(x)\right| \geq$ $|\langle x\rangle+Z(G)|=p^{3}$. If $\left|C_{G}(x)\right|=p^{4}$ then the result follows from Corllary 4.5 , so we take $\left|\operatorname{ker} \varphi_{x}\right|=\left|C_{G}(x)\right|=p^{3}$. But then $\left|\operatorname{Im} \varphi_{x}\right|=\left|G / \operatorname{ker} \varphi_{x}\right|=p^{2}$. Thus $|[x, G]|=p^{2}$, so $[x, G]=G^{\prime}$ which means $G^{\prime} \subseteq \bar{x}$ for each $x \notin Z(G)$. Thus, if $N \unlhd G$ and $N \nsubseteq Z(G)$ then $N \supseteq G^{\prime}$. This shows that $Z(G)$ cuts $\eta(G)$ and so $C_{0}(G)$ is not a ring.

In conclusion we have found that when $G$ is a finite abelian $p$-group then $C_{0}(G)$ is a ring if and only if $G$ is 1 -affine complete. For nonabelian $p$-groups, $p=2$, we have seen that $C_{0}(G)$ can be a ring properly containing $P_{0}(G)$. For $p>2$ we have several classes for which $C_{0}(G)$ is not a ring. In fact, for $p>2$ the authors have no example of a nonabelian $p$-group for which $C_{0}(G)$ is a ring unless $G$ is 1-affine complete. We thus close with the following.

Conjecture For finite nonabelian $p$-groups $G, p>2, C_{0}(G)$ is a ring if and only if $G$ is 1-affine complete.

Acknowledgements Open access funding provided by Austrian Science Fund (FWF). Portions of this research were done while the first author was visiting the Institute for Algebra at Johannes Kepler UniversitätLinz. He wishes to express his appreciation for the financial assistance and the gracious hospitality provided. Both authors wish to thank the Austrian Science Fund (FWF), Project FWF24077, for financial support.

Open Access This article is distributed under the terms of the Creative Commons Attribution 4.0 International License (http://creativecommons.org/licenses/by/4.0/), which permits unrestricted use, distribution, and reproduction in any medium, provided you give appropriate credit to the original author(s) and the source, provide a link to the Creative Commons license, and indicate if changes were made. 


\section{References}

1. Aichinger, E.: The near-ring of congruence preserving functions on an expanded group. J. Pure Appl. Algebra 205, 74-93 (2006)

2. Aichinger, E., Binder, F., Ecker, J., Mayr, P., Nöbauer, C.: SONATA—system of near-rings and their applications, GAP package, Version 2.6. http://www.algebra.uni-linz.ac.at/Sonata/. (2012)

3. Aichinger, E., Lazic, M., Mudrinski, N.: Finite generation of congruence preserving functions. Monatshefte für Mathematik 181, 35-62 (2015)

4. Aichinger, E., Mudrinski, N.: Types of polynomial completeness of expanded groups. Algebra Univers. 60(3), 309-343 (2009)

5. Aichinger, E., Mudrinski, N.: Sequences of commutator operations. Order 30(3), 859-867 (2013)

6. Berkovich, Y.: Groups of Prime Power Order. Vol. 1, Volume 46 of de Gruyter Expositions in Mathematics. Walter de Gruyter GmbH \& Co. KG, Berlin (2008). (With a foreword by Zvonimir Janko)

7. Burris, S., Sankappanavar, H.P.: A Course in Universal Algebra, Volume 78 of Graduate Texts in Mathematics. Springer, New York (1981)

8. Chandy, A.J.: Rings generated by the inner-automorphisms of non-abelian groups. Proc. Am. Math. Soc. 30, 59-60 (1971)

9. Cortini, R.: On special p-groups. Boll. Un. Math. Ital. 8, 677-689 (1998)

10. Ecker, J.: On the number of polynomial functions on nilpotent groups of class 2. Contrib. Gen. Algebra 10, 133-137 (1998)

11. Lausch, H., Nöbauer, W.: Algebra of Polynomials. North-Holland Mathematical Library, Vol. 5. NorthHolland Publishing Co., Amsterdam (1973)

12. Levi, F., van der Waerden, B.L.: Über eine besondere Klasse von Gruppen. Abh. Math. Semin. Univ. Hambg. 9(1), 154-158 (1933)

13. Levi, F.W.: Groups in which the commutator operation satisfies certain algebraic conditions. J. Indian Math. Soc. (N.S.) 6, 87-97 (1942)

14. Nöbauer, W.: Über die affin vollständigen, endlich erzeugbaren Moduln. Monatsh. Math. 82(3), 187198 (1976)

15. Quackenbush, R., Wolk, B.: Strong representation of congruence lattices. Algebra Univers. 1:165-166 $(1971 / 72)$

16. Robinson, D.J.S.: A Course in the Theory of Groups, Volume 80 of Graduate Texts in Mathematics, 2nd edn. Springer, New York (1996)

17. Saxinger, F.: Computer-assisted analysis of polynomial completeness of groups and rings. Master's thesis, JKU Linz (2013) 